\title{
Analysis of parallel diagonally implicit iteration of Runge-Kutta methods
}

\author{
P.J. van der Houwen and B.P. Sommeijer \\ Centre for Mathematics and Computer Science, P.O. Box 4079, 1009 AB Amsterdam. Netherlands
}

\begin{abstract}
Van der Houwen, P.J. and B.P. Sommeijer, Analysis of parallel diagonally implicit iteration of Runge-Kutta methods, Applied Numerical Mathematics 11 (1993) 169-188.

In this paper, we analyze parallel, diagonally implicit iteration of Runge-Kutta methods (PDIRK methods) for solving large systems of stiff equations on parallel computers. Like Newton-iterated backward differentiation formulas (BDFs), these PDIRK methods are such that in each step the (sequential) costs consist of solving a number of linear systems with the same matrix of coefficients and with the same dimension as the system of differential equations. Although for PDIRK methods the number of linear systems is usually higher than for Newton iteration of BDFs, the more computationally intensive work of computing the matrix of coefficients and its LU-decomposition are identical. The advantage of PDIRK methods over Newton-iterated BDFs is their unconditional stability (A-stability for Gauss-based methods and L-stability for Radau-based methods) for any order of accuracy.

Special characteristics of the PDIRK methods will be studied, such as the rate of convergence, the influence of particular predictors on the resulting stability properties, and the stiff error constants in the global error.
\end{abstract}

Keywords. Diagonally implicit Runge-Kutta methods; parallelism.

\section{Introduction}

Consider the initial value problem for systems of ordinary differential equations (ODEs) or dimension $d$

$$
\begin{aligned}
& y^{\prime}(t)=f(t, y(t)), \quad y\left(t_{0}\right)=y_{0}, \\
& y: \mathbb{R} \rightarrow \mathbb{R}^{d}, \quad f: \mathbb{R} \times \mathbb{R}^{d} \rightarrow \mathbb{R}^{d}, \quad t_{0} \leqslant t \leqslant t_{\mathrm{end}} .
\end{aligned}
$$

In this paper, we analyze integration methods based on iteration of implicit Runge-Kutta (RK) methods of collocation type. Such RK methods possess both a large step-point order and a large stage order. Furthermore, by a suitable choice of the collocation parameters, these RK methods are unconditionally stable for any order of accuracy.

We shall employ the diagonally implicit iteration-type methods proposed in [11,12]. These

Correspondence to: B.P. Sommeijer, Centre for Mathematics and Computer Science, P.O. Box 4079, 1009 AB Amsterdam, Netherlands. 
methods are designed in such a way that a large number of the implicit systems to be solved can be processed in parallel, so that the number of systems that have to be solved sequentially is substantially reduced when implemented on multi-processor computers. As a reference method, we take the method based on the backward differentiation formulas (BDFs), which is considered as one of the best methods for sequential computers. The sequential computations (i.e., the computations that cannot be performed in parallel on a multi-processor system) of the parallel diagonally implicitly iterated RK (PDIRK) methods are of the same nature as those of Newton-iterated BDFs, that is, in each step, both types of methods require the sequential solution of a number of linear systems with the same matrix of coefficients and with the same dimension as the system of differential equations. Although, this number of linear systems is usually higher for PDIRK methods than for Newton iteration of BDFs, the effort required for computing the Jacobian and the LU-decomposition of the matrix of coefficients is identical. For large systems of equations, these computations are the more computationally intensive work, so that the overall computation time is primarily determined by the number of Jacobian updates and LU-decompositions. The advantage of PDIRK methods over Newton-iterated BDFs is their A-stability (Gauss correctors [2]), strong A-stability (Lagrange correctors derived in [11]) or even L-stability (e.g. Radau IIA correctors) for high orders of accuracy. The property that unconditional stability can be combined with high orders reduces the number of integration steps (and therefore the number of Jacobian updates and LU-decompositions) considerably.

In Section 2, we define the PDIRK iteration scheme and discuss some favourable properties of the underlying implicit RK method (the corrector). We analyze the influence of the initial iterate (the predictor) with respect to the stability of the final result. Both implicit and explicit redictors of one-step and multistep type are discussed. Furthermore, several options for the .teration parameters are considered. Section 3 describes the convergence and stability for several predictor-corrector (PC) combinations. An expression for the global error for the linear inhomogeneous test equation $y^{\prime}(t)=\lambda y(t)+g(t)$ will be derived in Section 4. For various PC combinations and several one-step predictors, the principal stiff error constants in the global error expansion are calculated for several iteration strategies. Finally, in Section 5 the results are compared and some recommendations are formulated.

\section{PDIRK methods}

In this section we define PDIRK methods by specifying the RK corrector, the iteration scheme for solving the stage vector equation, the predictor formula, and the formula for the step-point values. The various families of PDIRK methods are determined by special choices of the iteration parameters occurring in the iteration scheme. In order to simplify the notations, the formulas are given for scalar ODEs. The extension to systems of ODEs is straightforward.

\subsection{The corrector}

We consider RK methods of the form

$$
\begin{aligned}
& Y-h A f\left(\boldsymbol{e t}_{n}+c h, \boldsymbol{Y}\right)=\boldsymbol{e} y_{n}+h \boldsymbol{a} f\left(t_{n}, y_{n}\right), \\
& y_{n+1}=y_{n}+h b_{0} f\left(t_{n}, y_{n}\right)+h \boldsymbol{b}^{\mathrm{T}} f\left(\boldsymbol{e} t_{n}+\boldsymbol{c h}, \boldsymbol{Y}\right), \quad \boldsymbol{c}:=\boldsymbol{a}+A \boldsymbol{e},
\end{aligned}
$$


where $b_{0}$ is a scalar parameter, $\boldsymbol{e}$ is the vector with unit entries, $\boldsymbol{a}=\left(a_{i}\right), \boldsymbol{b}=\left(b_{i}\right)$, and $\boldsymbol{c}=\left(c_{i}\right)$ are $k$-dimensional vectors, and $A=\left(a_{i j}\right)$ is a $k \times k$ matrix. In (2.1) we used the convention that for any given vectors $\boldsymbol{v}=\left(v_{j}\right)$ and $\boldsymbol{t}=\left(t_{j}\right), f(\boldsymbol{t}, \boldsymbol{v})$ denotes the vector with entries $f\left(t_{j}, v_{j}\right)$. We always assume that the matrix $A$ is nonsingular. If the vector $a$ or the parameter $b_{0}$ does not vanish, then (2.1) presents an $(s=k+1)$-stage RK method requiring $k$ implicit stages and one explicit stage. If $\boldsymbol{a}=\mathbf{0}$ and $b_{0}=0$, then (2.1) reduces to the general $(s=k)$-stage RK method with $s$ implicit stages. For a discussion of the order of accuracy and the stage order of RK methods, we refer to e.g. [4] and [3]. In the sequel, the method (2.1) will be called the corrector.

\subsection{The iteration scheme}

The stage vector equation in (2.1) is solved by applying the diagonal iteration method studied in $[11,12]$. Let $Y^{(\mu)}$ denote the successive iterates, then we may define the (highly parallel) iteration process

$$
\begin{aligned}
& \boldsymbol{Y}^{(1)}-h D f\left(\boldsymbol{e} t_{n}+c h, Y^{(1)}\right) \\
& \quad=\boldsymbol{e} y_{n}+\boldsymbol{a h f}\left(t_{n}, y_{n}\right)+h A f\left(e t_{n}+c^{*} h, Y^{(0)}\right)-h D f\left(e t_{n}+c^{*} h, Y^{(0)}\right), \\
& \boldsymbol{Y}^{(\mu)}-h D f\left(e t_{n}+c h, Y^{(\mu)}\right) \\
& \quad=e y_{n}+a h f\left(t_{n}, y_{n}\right)+h A f\left(e t_{n}+c h, Y^{(\mu-1)}\right)-h D f\left(e t_{n}+c h, Y^{(\mu-1)}\right),
\end{aligned}
$$

where $\mu=2, \ldots, m$, and $D$ is a diagonal matrix whose diagonal elements $\delta_{i}(i=1, \ldots, k)$ are the iteration parameters which are assumed to be positive. The parameter vector $c^{*}$ depends on the predictor formula used for computing $Y^{(0)}$ and serves to make the arguments of $f$ consistent in the first iteration (see Section 2.4). The step-point formula defining $y_{n+1}$ and the predictor formula will be discussed in the Sections 2.3 and 2.4, respectively. Together, the predictor formula, the iteration scheme (2.2), and the step-point formula determine the PDIRK method.

Each iteration in (2.2) requires the solution of $k$ nonlinear systems which can be obtained by applying modified Newton iteration. We shall call this last iteration the inner iteration method and the iteration (2.2) the outer iteration method. Notice that in each outer iteration the $k$ nonlinear systems can be solved in parallel, provided that $k$ processors are available. Thus, the sequential costs per step consist of computing $Y^{(0)}$ and of solving $m$ nonlinear systems of ODE dimension.

For particular choices of the predictor formula (e.g., explicit RK formulas) and for step-point formulas as defined in Section 2.3, the PDIRK method as described above can be interpreted as a diagonally implicit RK (DIRK) method using $m k$ diagonally implicit stages. Since the $k$ stages in each outer iteration can be computed in parallel, we arrive at a DIRK method with $m$ sequential diagonally implicit stages. These methods form a subclass of the much wider class of the PaRK methods investigated by Jackson and Nørsett $[7,8]$.

In $[11,12]$ the performance of PDIRK methods was studied in the case where in each of the $m$ outer iterations the inner iteration method was continued until convergence before starting the next outer iteration (this iteration strategy is also used in conventional DIRK methods). However, this strategy may be rather expensive if many iterations are needed to get the inner iteration converged. Moreover, it does not take into account the special structure of the 
method. The essential difference with conventional DIRK methods lies in the fact that the $i$ th component of each stage vector $Y^{(\mu)}$ is an approximation to the exact solution at the point $t_{n}+c_{i} h$. This implies that $Y^{(\mu-1)}$ furnishes an excellent initial approximation to the solution $\boldsymbol{Y}^{(\mu)}$ to be obtained in the inner iteration process. As a consequence, each outer iteration needs only a few inner iterations. Furthermore, in first approximation, the convergence of the inner-outer iteration scheme and the stability of the PDIRK method does not depend on the number of inner iterations. This motivates our strategy to perform only one inner iteration per outer iteration, leading to the iteration process

$$
\begin{aligned}
{[I-} & h D J]\left(Y^{(1)}-Y^{(1)}\right) \\
= & Y^{(1)}-h D f\left(e t_{n}+c h, Y^{(0)}\right) \\
& -\left[e y_{n}+a h f\left(t_{n}, y_{n}\right)+h A f\left(e t_{n}+c^{*} h, Y^{(0)}\right)-h D f\left(e t_{n}+c^{*} h, Y^{(0)}\right)\right], \\
& {[I-h D J]\left(Y^{(\mu-1)}-Y^{(\mu)}\right) } \\
= & Y^{(\mu-1)}-\left[e y_{n}+a h f\left(t_{n}, y_{n}\right)+h A f\left(e t_{n}+c h, Y^{(\mu-1)}\right)\right], \quad \mu=2, \ldots, m .
\end{aligned}
$$

Here, $J$ denotes an approximation to the derivative of $f$ at the point $\left(t_{n}, y_{n}\right)$. Evidently, if (2.3a) converges, then $Y^{(\mu)}$ converges to $Y$. In fact, one may interpret (2.3a) as a modified Newton iteration scheme for solving $Y$ from the stage vector equation in (2.1) employing a diagonal approximation to the Jacobian of $Y-h A f\left(e t_{n}+c h, Y\right)$.

It may be useful to consider (2.3a) in the case of systems of ODEs. Then, the $k$ components $Y_{i}^{(\mu)}$ of the stage vector iterate $Y^{(\mu)}$ have to satisfy the equations

$$
\begin{aligned}
& {\left[I-h \delta_{i} J\right]\left(Y_{i}^{(0)}-Y_{i}^{(1)}\right)} \\
& =Y_{i}^{(0)}-h \delta_{i} f\left(t_{n}+c_{i} h, Y_{i}^{(())}\right) \\
& -\left[\boldsymbol{y}_{n}+a_{i} h f\left(t_{n}, \boldsymbol{y}_{n}\right)+h \sum_{j=1}^{k} a_{i j} f\left(t_{n}+c_{j}^{*} h, \boldsymbol{Y}_{j}^{(0)}\right)-h \delta_{i} f\left(t_{n}+c_{i}^{*} h, \boldsymbol{Y}_{i}^{(0)}\right)\right], \\
& {\left[I-h \delta_{i} J\right]\left(Y_{i}^{(\mu-1)}-Y_{i}^{(\mu)}\right)} \\
& =Y_{i}^{(\mu-1)}-\left[\boldsymbol{y}_{n}+a_{i} h f\left(t_{n}, \boldsymbol{y}_{n}\right)+h \sum_{j=1}^{k} a_{i j} f\left(t_{n}+c_{j} h, \boldsymbol{Y}_{j}^{(\mu-1)}\right)\right], \quad \mu=2, \ldots, m,
\end{aligned}
$$

where $i=1, \ldots, k$ and where now $J$ denotes an approximation to the Jacobian matrix of $f$ at the point $\left(t_{n}, y_{n}\right)$. Notice that this iteration scheme can be viewed as a modified Newton method for solving the stage vector equation employing a block-diagonal approximation to the Jacobian. Clearly, the $k$ linear systems that are to be solved in each outer iteration step can be solved in parallel. Since each system has dimension equal to that of the system of ODEs, the computational complexity per step and per processor essentially consists of the computation of $Y_{i}^{(0)}$, the evaluation and LU-decomposition of the matrix $I-h \delta_{i} J$ (or its updating), $m+2$ evaluations of $f$, and $m$ forward-backward substitutions. Of these costs, the evaluation and LU-decomposition of $I-h \delta_{i} J$ are the most time-consuming, while the evaluations of $f$ and the forward-backward substitutions are relatively cheap (notice that the iteration parameters $\delta_{i}$ are independent of $\mu$ in order to avoid repeated LU-decompositions of $I-h \delta_{i} J$ in the 
successive iterations). Thus, when basing a code on PDIRK methods, first of all the number of stepsize changes (which automatically requires new LU-decompositions) and the number of Jacobian updates should be minimized.

It is of interest to compare the sequential costs of PDIRK methods with the sequential costs of the celebrated BDF-based methods. If the BDFs are solved by using $m$ modified Newton iterations, then the sequential costs in each step of the PDIRK methods and the Newton-iterated BDFs are almost identical. We expect that PDIRK methods need more iterations but, because of their higher order, less steps to produce some given accuracy. As explained above, evaluations of $f$ and the forward-backward substitutions are relatively cheap, so that for modest values of $m$, the sequential costs per step of PDIRK methods are expected to be not much higher than those of the BDFs. The reduced number of steps required by the PDIRKs should make them superior to the BDFs.

\subsection{The step-point values}

Suppose that we adopt $Y^{(m)}$ as a sufficiently accurate approximation to the exact stage vector solution $\boldsymbol{Y}$ of the corrector (2.1). Then, the most natural way to approximate the step-point value $y_{n+1}$ in (2.1) defines this value according to the formula

$$
y_{n+1}=y_{n}+h b_{0} f\left(t_{n}, y_{n}\right)+h \boldsymbol{b}^{\mathrm{T}} f\left(\boldsymbol{e} t_{n}+c h, \boldsymbol{Y}^{(m)}\right) .
$$

However, the presence of the right-hand side evaluations in this formula may give rise to loss of accuracy in the case of stiff problems (cf. [10]). This difficulty can be overcome by applying . similar approach as proposed in [6] for the implementation of implicit RK methods. Observi that the corrector (2.1) can be written in the form

$$
y_{n+1}=y_{n}+b_{0} h f\left(t_{n}, y_{n}\right)+\boldsymbol{b}^{\mathrm{T}} A^{-1}\left[\boldsymbol{Y}-\boldsymbol{e} y_{n}-\boldsymbol{a h f}\left(t_{n}, y_{n}\right)\right],
$$

provided that $A$ is nonsingular, we can approximate the corrector solution $y_{n+1}$ by the formula

$$
y_{n+1}=y_{n}+b_{0} h f\left(t_{n}, y_{n}\right)+\boldsymbol{b}^{\mathrm{T}} A^{-1}\left[\boldsymbol{Y}^{(m)}-\boldsymbol{e} y_{n}-\boldsymbol{a h f}\left(t_{n}, y_{n}\right)\right],
$$

where $\boldsymbol{Y}^{(m)}$ denotes the last computed approximation to $\boldsymbol{Y}$. In many cases the corrector satisfies the relations of stiff accuracy, i.e., $c_{k}=1, b_{0}=a_{k}$, and $\boldsymbol{b}^{\mathrm{T}} A^{-1}=\boldsymbol{e}_{k}^{\mathrm{T}}$, so that (2.3b) reduces to $y_{n+1}=\boldsymbol{e}_{k}^{\mathrm{T}} \boldsymbol{Y}^{(m)}$. In order to avoid confusion, we shall from now on denote the corrector solution and stage vector values obtained from $y_{n}$ by $u_{n+1}$ and $\boldsymbol{U}$, respectively.

\subsection{The predictor}

In [12] we considered one-step predictors of the form

$$
\boldsymbol{Y}^{(1)}:=\boldsymbol{e} y_{n}+h E f\left(\boldsymbol{e} t_{n}, \boldsymbol{e} y_{n}\right)+h B f\left(\boldsymbol{e} t_{n}+\boldsymbol{c}^{*} h, Y^{(0)}\right),
$$

where $B$ and $E$ are $k \times k$ matrices. Of particular interest are the cases where $E$ vanishes and where $B$ is either the zero matrix yielding last step-value predictors (LSP) or $B=D$ yielding implicit Euler predictors (IEP).

However, by using information from the preceding step, that is the values of $y_{n}$ and the stage vector $\boldsymbol{Y}^{(m)}$ computed in the last step, we can construct more accurate predictors. In order to 
indicate to which step a particular stage vector corresponds, we define $Y_{n}:=\boldsymbol{Y}^{(m)}$ if $\boldsymbol{Y}^{(m)}$ corresponds to the step $\left[t_{n-1}, t_{n}\right]$. Consider the two-step predictor

$$
\boldsymbol{Y}^{(0)}=V \boldsymbol{Y}_{n}+v y_{n}+h B f\left(e t_{n}+c^{*} h, Y^{(0)}\right),
$$

where either $B=O$ or $B=D$, and where the matrix $V$ and the vector $v$ satisfy the usual consistency conditions (we shall assume that the vector $v$ vanishes in the case of stiffly accurate correctors). The cases $B=O$ and $B=D$ will be referred to as the extrapolation predictor (EXP) and the backward differentiation predictor (BDP).

If $B=D$, then both (2.5) and (2.6) require the solution of $k$ implicit relations. Similar to the strategy followed in solving the implicit relations in (2.2), we shall perform just one Newton iteration. (Notice that the right-hand side derivatives required in the Newton iteration method are identical to those occurring in (2.3a).) In order to perform this Newton iteration we need an initial guess $Y^{(-1)}$ for $Y^{(0)}$. For the cases (2.5) and (2.6) we shall respectively use

$$
\begin{aligned}
& Y^{(-1)}=\boldsymbol{e} y_{n}, \quad \boldsymbol{c}^{*}=(E+B) \boldsymbol{e}, \\
& \boldsymbol{Y}^{(-1)}=W \boldsymbol{Y}_{n}+\boldsymbol{w} y_{n}, \quad \boldsymbol{c}^{*}=\boldsymbol{c},
\end{aligned}
$$

where the $W$ and $\boldsymbol{w}$ are to be determined (we shall assume that $\boldsymbol{w}$ vanishes in the case of stiffly accurate correctors, and that $W=V$ and $\boldsymbol{w}=\boldsymbol{v}$ in the case where $B=O)$ ). If the corrector is based on collocation, then the matrix $W$ and the vector $w$ can be computed by extrapolating the collocation polynomial defined in $\left[t_{n-1}, t_{n}\right]$ to the interval $\left[t_{n}, t_{n+1}\right]$ and can be expressed in terms of the Lagrange interpolation polynomials.

\subsection{The iteration parameters}

There are various options for choosing the number of iterations $m$, and the iteration parameters $\delta_{i}$. In this paper, we consider three cases:

Option 1 ( fixed-number-of-iterations option):

- the number of iterations is fixed and such that the orders of the PDIRK and corrector are equal;

- the iteration parameters are chosen such that the stability region in the left halfplane is optimized.

Option 2 (minimal-spectral-radius option):

- the number of iterations is sufficiently large to closely approximate the corrector solution;

- the iteration parameters are such that the spectral radius of the matrix $D^{-1} A-I$ is minimized.

Option 3 (minimal-stiff-error-constant option):

- the number of iterations is sufficiently large to closely approximate the corrector solution;

- the iteration parameters are such that the principal stiff error constant of the PDIRK method is minimized.

Several families of methods constructed according to the fixed-number-of-iterations option were already considered in [12]. An interesting family considered in that paper possesses the stability functions investigated by Wolfbrandt [13] and uses constant iteration parameters $\delta_{i}$ determined by these stability functions. However, because of the fixed number of iterations, 
these methods are in fact DIRK methods and consequently, they have the disadvantage of possessing stage order $q=1$. In many stiff problems, such a low stage order may lead to reduced accuracies. In order to get insight into the extent of this accuracy reduction, we shall consider the magnitude of the stiff error constants for the "fixed-number-of-iterations PDIRK methods" (see Section 4.2, Table 4).

For the explicit one-step predictor, [11] presents a number of PDIRK methods constructed according to the minimal-spectral-radius option. The effect of minimizing the spectral radius of the matrix $D^{-1} A-I$ is a strong damping of the stiff iteration error components. On the one hand, the number of iterations $m$ should be sufficiently large to solve more or less the RK corrector, on the other hand, $m$ should be sufficiently small to achieve that the (sequential) costs per step are not excessive when compared with those of the BDFs. In this paper, we shall investigate a few characteristics of the "minimal-spectral-radius PDIRK methods" as a function of $m$. In particular, in Section 3 we consider the rate of convergence (Table 1) and the effect on the stability of the various predictors (Tables 2 and 3), and in Section 4 we consider the magnitude of the principal stiff error constants (Tables 5 and 6).

Option 3 offers an alternative to option 2 and directly addresses the truncation error of PDIRK methods when applied to stiff systems. In this paper, we present results for the simple inhomogeneous test equation $y^{\prime}(t)=\lambda y(t)+g(t)$.

\section{Convergence and stability}

We shall investigate convergence and stability by means of the scalar test equation $y^{\prime}=\lambda y$. Note that for this simple test equation the particular strategy used in the inner iterations is not relevant. For a rigorous convergence analysis of parallel RK methods containing the PDIRK methods of this paper we refer to Jackson and Nørsett $[7,8]$.

\subsection{Rate of convergence}

From (2.2) it can be deduced that the iteration error satisfies the recursion

$$
\begin{aligned}
& U-Y_{n+1}=Z(z)\left(\boldsymbol{U}-\boldsymbol{Y}^{(m-1)}\right)=\cdots=Z^{m}(z)\left(\boldsymbol{U}-\boldsymbol{Y}^{(0)}\right), \\
& Z(z):=z D[I-z D]^{-1}\left[D^{-1} A-I\right], \quad z:=\lambda h .
\end{aligned}
$$

The region in the complex $z$-plane where $Z^{m}(z) \rightarrow 0$ for $m \rightarrow \infty$ will be called the region of convergence. We define the iteration function $C$ of the PDIRK method by the spectral radius of $Z(z)$, i.e.,

$$
C(z):=\rho(Z(z))=\rho\left(z D[I-z D]^{-1}\left[D^{-1} A-I\right]\right) .
$$

Evidently, the region of convergence is determined by the set of points where $C(z)<1$. The rate of convergence is larger as the norm of $C(z)$ is smaller in the region of relevant values of $z$. Thus, adopting the maximum norm, we are led to the minimization of $C(z)$ in this region. In this connection we introduce the following definition: 
Definition 3.1. A PDIRK method is said to be strongly A-convergent if its iteration function $C(z) \leqslant \eta<1$ in the whole left halfplane $\operatorname{Re} z<0$. If, in addition, $C(-\infty)=0$, then the PDIRK method is called L-convergent.

First we consider the constant $-\delta_{i}$ case which is of interest in the case of fixed-number-of-iterations methods.

Theorem 3.2. If $D$ has constant, positive diagonal elements, then minimization of $\rho\left(D^{-1} A-I\right)$ implies that the norm of $C(z)$ is minimized whenever $z$ is in the left halfplane.

Proof. If $D=\delta \cdot I$, then we may write $C(z)=|\delta z| \rho\left(\delta^{-1} A-I\right) /|1-\delta z|$. In the left halfplane, the maximum of the function $|\delta z /(1-\delta z)|$ does not depend on $\delta$, provided that $\delta>0$. Hence, the norm of $C(z)$ is minimized if $\rho\left(D^{-1} A-I\right)$ is minimized.

In the case where $D$ does not have constant diagonal entries, we cannot derive such a simple expression for $C(z)$, and a numerical search is needed to find the matrix $D$ that minimizes the norm of $C(z)$ in the left halfplane. However, our numerical experiments revealed that also in the nonconstant $-\delta_{i}$ case the minimization of $\rho\left(D^{-1} A-I\right)$ yields fast converging PDIRK methods and that $\|C\|:=\max \{C(z): \operatorname{Re} z \leqslant 0\}$ is considerably smaller than in the constant $\delta_{i}$ case.

Example 3.3. We consider an example of the fixed-number-of-iterations methods studied in [12] which is based on the third-order Radau IIA corrector. For

$$
m=3, \quad A=\frac{1}{12}\left(\begin{array}{cc}
5 & -1 \\
9 & 3
\end{array}\right), \quad D=\delta \cdot I, \quad \delta=0.43586650,
$$

this leads to a third-order, L-stable PDIRK method. The convergence function associated with this method is given by

$$
C(z)=|\delta z| \rho\left(\delta^{-1} A-I\right) /|1-\delta z|,
$$

vhere

$$
\rho\left(\delta^{-1} A-I\right)=\delta^{-1} \sqrt{1 / 6-2 \delta / 3+\delta^{2}} .
$$

tting $\delta=0.43586650$ we find that $C(z)<0.59$ in the whole left halfplane. Among the methods with $D=\delta \cdot I$ this method is almost optimal (the minimizing value is given by $\delta=1 / 2$ leading to $C(z)<\sqrt{1 / 3} \approx 0.577$ ).

Next, we consider the case where $D$ minimizes $\rho\left(D^{-1} A-I\right)$. In [11] it was shown that the method can be made L-convergent (i.e., it has vanishing $\rho\left(D^{-1} A-I\right)$ ) for $\delta_{1}=(4-\sqrt{6}) / 6$ and $\delta_{2}=(4+\sqrt{6}) / 10$. The corresponding matrix $Z(z)$ is easily computed, yielding $\|C\| \approx 0.262$.

Table 1 lists the $\|C\|$-values for a number of minimal-spectral-radius PDIRK methods. These methods are based on Radau IIA correctors and on the so-called Lagrange correctors derived in [11]. The Lagrange methods are strongly A-stable, stiffly accurate collocation methods which are completely determined by the collocation vector $c$ (see Table 1). Their stage order is one higher than that of the Radau IIA methods which was achieved by using one 
Table 1

$\|C\|$-values for minimal-spectral-radius PDIRK methods based on Radau IIA and Lagrange correctors

\begin{tabular}{|c|c|c|c|c|c|}
\hline Corrector & $k$ & & $\|C\|$ & $\begin{array}{l}\text { Strongly } \\
\text { A-convergent }\end{array}$ & L-convergent \\
\hline Radau IIA & $\begin{array}{l}2 \\
3 \\
4\end{array}$ & & $\begin{array}{l}0.262 \\
0.401 \\
0.527\end{array}$ & $\begin{array}{l}\text { yes } \\
\text { yes } \\
\text { yes }\end{array}$ & $\begin{array}{l}\text { yes } \\
\text { no } \\
\text { no }\end{array}$ \\
\hline Lagrange & $\begin{array}{l}2 \\
3 \\
4\end{array}$ & $\begin{array}{l}c=(3 / 4,1)^{\mathrm{T}} \\
c=(7 / 12,5 / 6,1)^{\mathrm{T}} \\
c=(1 / 6,7 / 12,11 / 12,1)^{\mathrm{T}}\end{array}$ & $\begin{array}{l}0.182 \\
0.403 \\
0.404\end{array}$ & $\begin{array}{l}\text { yes } \\
\text { yes } \\
\text { yes }\end{array}$ & $\begin{array}{l}\text { yes } \\
\text { no } \\
\text { no }\end{array}$ \\
\hline
\end{tabular}

explicit and $k$ implicit stages. However, they do not possess the superconvergence property of the Radau methods, so that the computation of the nonstiff solution components is considerably less accurate.

For the Radau IIA and Lagrange correctors with $k$ implicit stages, the iteration parameters are contained in the matrices $D_{k \mathrm{R}}$ and $D_{k \mathrm{~L}}$ :

$$
\begin{aligned}
& D_{2 \mathrm{R}}=\frac{1}{30}\left(\begin{array}{cc}
20-5 \sqrt{6} & 0 \\
0 & 12+3 \sqrt{6}
\end{array}\right), \quad D_{2 \mathrm{~L}}=\left(\begin{array}{cc}
\frac{3}{4(\sqrt{2}+1)} & 0 \\
0 & \frac{1}{6(\sqrt{2}-1)}
\end{array}\right) \text {, } \\
& D_{3 \mathrm{R}}=\left(\begin{array}{ccc}
\frac{4.365}{1.3624} & 0 & 0 \\
0 & \frac{10.32}{7373} & 0 \\
0 & 0 & \frac{1887}{5077}
\end{array}\right), \quad D_{3 \mathrm{~L}}=\left(\begin{array}{ccc}
\frac{2246}{10669} & 0 & 0 \\
0 & \frac{2537}{8794} & 0 \\
0 & 0 & \frac{3026}{8923}
\end{array}\right) \text {, } \\
& D_{4 \mathrm{R}}=\left(\begin{array}{cccc}
\frac{3055}{9532} & 0 & 0 & 0 \\
0 & \frac{531}{5956} & 0 & 0 \\
0 & 0 & \frac{1471}{8094} & 0 \\
0 & 0 & 0 & \frac{1848}{7919}
\end{array}\right), \quad D_{4 \mathrm{~L}}=\left(\begin{array}{cccc}
\frac{5147}{38467} & 0 & 0 & 0 \\
0 & \frac{1983}{17459} & 0 & 0 \\
0 & 0 & \frac{3197}{14090} & 0 \\
0 & 0 & 0 & \frac{3086}{12339}
\end{array}\right) \text {. }
\end{aligned}
$$

Table 1 shows that these methods can all be made strongly A-convergent, and that only the methods based on a two-stage corrector are L-convergent (see also [11]). Furthermore, we observe that the convergence factors of the Lagrange-based methods are slightly better. Hence, together with their increased stage order, the Lagrange correctors seem to be attractive alternatives to the Radau correctors in problems where the order of accuracy is determined by the stage order. However, in problems where, apart from the stage order, the nonstiff (or, classical) order is important, the superconvergent Radau correctors are to be preferred. As to the $\|C\|$-values given in Table 1 , it should be remarked that these are "worst-case" values, that is, in actual computation, where the relevant values of $z$ are located in a restricted region of the left halfplane, the corresponding bound on $C(z)$ may be much smaller. 


\subsection{Region of stability}

In order to investigate the stability properties of PDIRK methods we have to specify the predictor formula. The stability of PDIRK methods using the one-step predictor (2.5) was extensively discussed in [12] for the case where $y_{n+1}$ is defined by (2.4). For the case (2.3b) considered in this paper, we have the following theorems:

Theorem 3.4. For the equation $y^{\prime}=\lambda y$ the PDIRK solution generated by $\{(2.3 \mathrm{a}),(2.3 \mathrm{~b}),(2.5)\}$ satisfies the recursion

$$
y_{n+1}=R_{m}(z) y_{n}, \quad R_{m}(z):=R(z)-E_{m}(z),
$$

where

$$
\begin{aligned}
& R(z):=1+z b_{0}+z \boldsymbol{b}^{\mathrm{T}}[I-z A]^{-1}[\boldsymbol{e}+z \boldsymbol{a}] . \\
& E_{m}(z):=\boldsymbol{b}^{\mathrm{T}} A^{-1} Z^{m}(z)\left([I-z A]^{-1}[\boldsymbol{e}+z \boldsymbol{a}]-[I-z B]^{-1}[I+z E] \boldsymbol{e}\right) .
\end{aligned}
$$

Here, $R(z)$ is the stability function of the corrector reducing to

$$
R(z)=\boldsymbol{e}_{k}^{\mathrm{T}}[I-z A]^{-1}[\boldsymbol{e}+z \boldsymbol{a}]
$$

in the stiffly accurate case.

Proof. From the relations

$$
\boldsymbol{Y}^{(0)}=[I-z B]^{-1}[I+z E] e y_{n}, \quad U=[I-z A]^{-1}[e+z a] y_{n},
$$

it follows that

$$
\begin{aligned}
\boldsymbol{U}-\boldsymbol{Y}_{n+1} & =Z^{m}(z)\left(\boldsymbol{U}-\boldsymbol{Y}^{(0)}\right) \\
& =Z^{m}(z)\left([I-z A]^{-1}[\boldsymbol{e}+z \boldsymbol{a}]-[I-z B]^{-1}[I+z E] \boldsymbol{e}\right) y_{n} .
\end{aligned}
$$

Hence, from the step-point formula (2.3b) we obtain

$$
\begin{aligned}
u_{n+1}-y_{n+1} & =\boldsymbol{b}^{\mathrm{T}} A^{-1}\left(\boldsymbol{U}-\boldsymbol{Y}_{n+1}\right) \\
& =\boldsymbol{b}^{\mathrm{T}} A^{-1} Z^{m}(z)\left([I-z A]^{-1}[\boldsymbol{e}+z \boldsymbol{a}]-[I-z B]^{-1}[I+z E] \boldsymbol{e}\right) y_{n} .
\end{aligned}
$$

Furthermore, introducing the stability function $R(z)$ of the corrector, we may write

$$
u_{n+1}=R(z) y_{n}
$$

where $R(z)$ is defined in the theorem. From (3.6) and (3.7) the assertion of the theorem is immediate.

Theorem 3.5. For the equation $y^{\prime}=\lambda y$ the PDIRK solution generated by $\{(2.3),(2.6)\}$ satisfies the recursion

$$
\left(\begin{array}{l}
Y_{n+1} \\
y_{n+1}
\end{array}\right)=M_{m}(z)\left(\begin{array}{l}
Y_{n} \\
y_{n}
\end{array}\right)
$$


where $M_{m}(z)$ is the amplification matrix

$$
\begin{aligned}
M_{m}(z):= & \left(\begin{array}{cc}
I & \mathbf{0} \\
-\boldsymbol{b}^{\mathrm{T}} A^{-1} & 1
\end{array}\right)^{-1} \\
& \times\left(\begin{array}{cc}
Z^{m}(z)[I-z B]^{-1} V & {\left[I-Z^{m}(z)\right][I-z A]^{-1}[\boldsymbol{e}+z \boldsymbol{a}]+Z^{m}(z)[I-z B]^{-1} \boldsymbol{v}} \\
\mathbf{0}^{\mathrm{T}} & 1+b_{0} z-\boldsymbol{b}^{\mathrm{T}} A^{-1}[\boldsymbol{e}+z \boldsymbol{a}]
\end{array}\right) .
\end{aligned}
$$

Proof. By means of the equation for $\boldsymbol{U}$ given in (3.4), relation (3.5) and

$$
\boldsymbol{Y}^{(0)}=[I-z B]^{-1}\left[V \boldsymbol{Y}_{n}+v y_{n}\right]
$$

we derive that

$$
\begin{aligned}
\boldsymbol{Y}_{n+1}= & Z^{m}(z)[I-z B]^{-1} V \boldsymbol{Y}_{n} \\
& +\left(\left[I-Z^{m}(z)\right][I-z A]^{-1}[\boldsymbol{e}+z \boldsymbol{a}]+Z^{m}(z)[I-z B]^{-1} \boldsymbol{v}\right) y_{n} .
\end{aligned}
$$

Together with the step-point formula (2.3b) the one-step recursion of the theorem is easily obtained.

With the amplification matrix $M_{m}(z)$ we associate the stability function

$$
R_{m}(z):=\rho\left(M_{m}(z)\right)
$$

where $\rho\left(M_{m}\right)$ denotes the spectral radius of the matrix $M_{m}$. The region in the complex $z$-plane where $R_{m}(z)<1$ will be called the region of stability associated with $m$. Furthermore, we define $m_{\text {crit }}$ as the minimal value of $m$ for which this region contains the whole left halfplane for all $m \geqslant m_{\text {crit }}$.

For future reference, we have computed the value of $m_{\text {crit }}$ for a number of predictor-corrector (PC) pairs. For the correctors we again chose the Radau IIA methods and the Lagrange methods of Section 3.1. The predictors are those defined in Section 2.4 and the matrices $D$ are defined according to the minimal-spectral-radius option (see (3.3a), (3.3b), and (3.3c)). Table 2 shows that $m_{\text {crit }}$ increases if the number of stages of the corrector increases. However, in actual computation, the minimal number of iterations may be much smaller because many stiff problems require only $\mathrm{A}(\alpha)$-stability. This means that automatic codes based on PDIRK

Table 2

Values of $m_{\text {crit }}$ of minimal-spectral-radius PDIRK methods for various PC pairs

\begin{tabular}{llllll}
\hline Corrector & & LSP & EXP & IEP & BDP \\
\hline Radau IIA & $k=2$ & 1 & 1 & 1 & 1 \\
& $k=3$ & 5 & 5 & 2 & 4 \\
Lagrange & 7 & 7 & 4 & 7 & 2 \\
& $k=4$ & 2 & 3 & 2 & 3 \\
& $k=3$ & 3 & 7 & 3 & 6 \\
\hline
\end{tabular}


Table 3

Values of $\alpha=\alpha(m)$ (in degrees) of minimal-spectral-radius PDIRK methods for various PC pairs

\begin{tabular}{|c|c|c|c|c|c|c|c|c|c|}
\hline Predictor & Corrector & $k$ & $m=1$ & $m=2$ & $m=3$ & $m=4$ & $m=5$ & $m=6$ & $m=7$ \\
\hline $\begin{array}{l}\text { LSP } \\
\text { EXP } \\
\text { IEP } \\
\text { BDP }\end{array}$ & Radau IIA & 2 & $\begin{array}{l}90 \\
90 \\
90 \\
90\end{array}$ & & & & & & \\
\hline $\begin{array}{l}\text { LSP } \\
\text { EXP } \\
\text { IEP } \\
\text { BDP }\end{array}$ & & 3 & $\begin{array}{l}* \\
* \\
87.5 \\
65.0\end{array}$ & $\begin{array}{l}* \\
* \\
90 \\
81.8\end{array}$ & $\begin{array}{l}81.9 \\
64.7 \\
88.4\end{array}$ & $\begin{array}{l}89.94 \\
88.7 \\
90\end{array}$ & $\begin{array}{l}90 \\
90\end{array}$ & & \\
\hline $\begin{array}{l}\text { LSP } \\
\text { EXP } \\
\text { IEP } \\
\text { BDP }\end{array}$ & & 4 & $\begin{array}{l}* \\
* \\
60.2 \\
43.0\end{array}$ & $\begin{array}{l}* \\
* \\
75.9 \\
14.6\end{array}$ & $\begin{array}{l}* \\
* \\
86.1 \\
67.1\end{array}$ & $\begin{array}{l}40.3 \\
* \\
90 \\
78.2\end{array}$ & $\begin{array}{l}80.5 \\
70.3 \\
84.6\end{array}$ & $\begin{array}{l}88.5 \\
84.2 \\
88.6\end{array}$ & $\begin{array}{l}90 \\
90 \\
90\end{array}$ \\
\hline $\begin{array}{l}\text { LSP } \\
\text { EXP } \\
\text { IEP } \\
\text { BDP }\end{array}$ & Lagrange & 2 & $\begin{array}{l}* \\
* \\
86.5 \\
89.82\end{array}$ & $\begin{array}{l}90 \\
90 \\
90 \\
90\end{array}$ & & & & & \\
\hline $\begin{array}{l}\text { LSP } \\
\text { EXP } \\
\text { IEP } \\
\text { BDP }\end{array}$ & & 3 & $\begin{array}{l}* \\
* \\
77.2 \\
83.4\end{array}$ & $\begin{array}{l}* \\
* \\
* \\
*\end{array}$ & $\begin{array}{l}90 \\
90 \\
90 \\
90\end{array}$ & & & & \\
\hline $\begin{array}{l}\text { LSP } \\
\text { EXP } \\
\text { IEP } \\
\text { BDP }\end{array}$ & & 4 & $\begin{array}{l}* \\
* \\
51.6 \\
48.8\end{array}$ & $\begin{array}{l}* \\
* \\
* \\
*\end{array}$ & $\begin{array}{l}* \\
* \\
* \\
*\end{array}$ & $\begin{array}{l}60.8 \\
* \\
86.5 \\
79.9\end{array}$ & $\begin{array}{l}86.7 \\
73.0 \\
90 \\
87.6\end{array}$ & $\begin{array}{l}90 \\
88.0 \\
90\end{array}$ & 90 \\
\hline
\end{tabular}

whe

met

methods are likely to choose the number of iterations not larger than necessary to ensure a stable performance. Table 3 presents the corresponding angles $\alpha$ as a function of $m$ (lack of $\mathrm{A}(0)$-stability is indicated by *). The results illustrate the favourable $\mathrm{A}(\alpha)$-stability characterisis of minimal-spectral-radius PDIRK methods after only a few iterations. In general, the ,plicit predictors IEP and BDP possess (of course) larger stability angles $\alpha$ than the explicit redictors LSP and EXP, even if we take into account that the implicit predictors require extra computational effort roughly comparable with an additional iteration. Furthermore, if we compare IEP and BDP, then IEP has the best stability characteristics (in particular for Radau-based methods). However, the overall efficiency will be reduced because of its low order of accuracy. Therefore, we drop the low-order predictors LSP and IEP and recommend either the EXP or BDP predictor.

\section{The error functions for the linear inhomogeneous test equation}

The following theorem presents a result for general RK methods derived in [1]: 
Theorem 4.1. For RK methods the global error $e_{n}$ when applied to the test equation $y^{\prime}(t)=\lambda y(t)$ $+g(t)$ satisfies

$$
\begin{aligned}
& e_{n+1}=R(z) e_{n}+\sum_{j=q+1} Q_{j}(z) h^{j} y_{\mathrm{ex}}^{(j)}\left(t_{n}\right), \\
& Q_{j}(z):=\frac{1}{j !}\left[1-j \boldsymbol{b}^{\mathrm{T}} \boldsymbol{c}^{j-1}\right]+\frac{1}{j !} z \boldsymbol{b}^{\mathrm{T}}[I-z A]^{-1}\left[\boldsymbol{c}^{j}-j A \boldsymbol{c}^{j-1}\right],
\end{aligned}
$$

where $y_{\mathrm{ex}}(t)$ denotes the exact solution of the test equation, $R(z)$ is the stability function of the $R K$ method, and $q$ is its stage order, i.e., the largest integer such that

$$
1-j \boldsymbol{b}^{\mathrm{T}} \boldsymbol{c}^{j-1}=\boldsymbol{c}^{j}-j A \boldsymbol{c}^{j-1}=0 \quad \text { for } j=1, \ldots, q .
$$

We shall prove a similar theorem for PDIRK methods employing one-step predictors. As before, the simplicity of the test equation $y^{\prime}(t)=\lambda y(t)+g(t)$ implies that the particular strategy used in the inner iteration process is not relevant.

In the following, $y(t)$ denotes the locally exact solution at $t_{n}$, i.e., $y_{n}=y\left(t_{n}\right)$. It is straightforwardly verified that for the linear inhomogeneous equation the recursion (3.5) changes to

$$
\boldsymbol{U}-\boldsymbol{Y}_{n+1}=Z^{m}(z)\left(\boldsymbol{U}-\boldsymbol{Y}^{(0)}+h z^{-1}\left[g\left(t_{n} \boldsymbol{e}+h \boldsymbol{c}\right)-g\left(t_{n} \boldsymbol{e}+h \boldsymbol{c}^{*}\right)\right]\right) .
$$

Assuming that $g$ is sufficiently differentiable, we may write for any fixed vector $v$

$$
g\left(t_{n} \boldsymbol{e}+h \boldsymbol{v}\right)=\sum_{j=0} \frac{1}{j !}(h \boldsymbol{v})^{j} g^{(j)}\left(t_{n}\right)=\frac{1}{h} \sum_{j=0} \frac{1}{j !} h^{j} y^{(j)}\left(t_{n}\right)\left[j \boldsymbol{v}^{j-1}-z \boldsymbol{v}^{j}\right] .
$$

Hence,

$$
\begin{aligned}
& h\left[g\left(t_{n} \boldsymbol{e}+h \boldsymbol{c}\right)-g\left(t_{n} \boldsymbol{e}+h \boldsymbol{c}^{*}\right)\right]=\sum_{j=1} \frac{1}{j !} \gamma_{j}(z) h^{j} y^{(j)}\left(t_{n}\right), \\
& \gamma_{j}(z):=j \boldsymbol{c}^{j-1}-z \boldsymbol{c}^{j}-j\left(\boldsymbol{c}^{*}\right)^{j-1}+z\left(\boldsymbol{c}^{*}\right)^{j} .
\end{aligned}
$$

Furthermore, it follows from (2.1) that

$$
\boldsymbol{U}=[I-z A]^{-1}\left[y\left(t_{n}\right) \boldsymbol{e}+h y^{\prime}\left(t_{n}\right) \boldsymbol{a}+h A g\left(t_{n} \boldsymbol{e}+h c\right)\right],
$$

so that

$$
\begin{aligned}
& \boldsymbol{U}=y_{n} \boldsymbol{e}+\sum_{j=1} \frac{1}{j !} \boldsymbol{c}_{j}(z) h^{j} y^{(j)}\left(t_{n}\right), \\
& \boldsymbol{c}_{1}(z):=\boldsymbol{c}, \quad \boldsymbol{c}_{j}(z):=[I-z A]^{-1} A\left[j \boldsymbol{c}^{j-1}-z \boldsymbol{c}^{j}\right], \quad j \geqslant 2 .
\end{aligned}
$$

\subsection{One-step predictors}

Let us assume that $\boldsymbol{Y}^{(0)}$ is provided by a one-step formula, then it can also be expanded in terms of a similar Taylor series with coefficients $c_{j}^{*}(z)$ :

$$
\boldsymbol{Y}^{(0)}=y_{n} \boldsymbol{e}+\sum_{j=1} \frac{1}{j !} c_{j}^{*}(z) h^{j} y^{(j)}\left(t_{n}\right) .
$$


Thus,

$$
\begin{aligned}
& U-Y_{n+1}=Z^{m}(z) \sum_{j=1} q_{j}(z) h^{j} y^{(j)}\left(t_{n}\right), \\
& q_{1}(z):=c^{*}-c_{1}^{*}(z), \quad q_{j}(z):=\frac{1}{j !}\left[c_{j}(z)-c_{j}^{*}(z)+z^{-1} \gamma_{j}(z)\right], \quad j \geqslant 2 .
\end{aligned}
$$

Assuming that $c_{1}^{*}(z)$ does not depend on $z$, we may choose in $(2.3) c^{*}=c_{1}^{*}$ so that $q_{1}(z)$ vanishes. Using the relation

$$
y^{(\prime)}\left(t_{n}\right)=y_{\mathrm{ex}}^{(j)}\left(t_{n}\right)+\lambda^{j}\left[y\left(t_{n}\right)-y_{\mathrm{ex}}\left(t_{n}\right)\right]=y_{\mathrm{ex}}^{(j)}\left(t_{n}\right)+\lambda^{j}\left[y_{n}-y_{\mathrm{ex}}\left(t_{n}\right)\right]
$$

the iteration error (4.4) can be expanded in terms of derivatives of the exact solution. We obtain

$$
\boldsymbol{U}-\boldsymbol{Y}_{n+1}=Z^{m}(z) \sum_{j=2} q_{j}(z)\left(z^{j}\left[y_{n}-y_{\mathrm{ex}}\left(t_{n}\right)\right]+h^{j} y_{\mathrm{ex}}^{(j)}\left(t_{n}\right)\right)
$$

Since

$$
u_{n+1}-y_{n+1}=\boldsymbol{b}^{\mathrm{T}} A^{-1}\left[\boldsymbol{U}-\boldsymbol{Y}_{n+1}\right],
$$

we find

$$
\begin{aligned}
& u_{n+1}-y_{n+1}=S_{m}(z)\left[y_{n}-y_{\mathrm{ex}}\left(t_{n}\right)\right]+\sum_{j=2} Q_{m j}(z) h^{j} y_{\mathrm{ex}}^{(j)}\left(t_{n}\right), \\
& S_{m}(z):=\boldsymbol{b}^{\mathrm{T}} A^{-1} Z^{m}(z) \sum_{j=2} \boldsymbol{q}_{j}(z) z^{j}, \quad Q_{m j}(z):=\boldsymbol{b}^{\mathrm{T}} A^{-1} Z^{m}(z) \boldsymbol{q}_{j}(z) .
\end{aligned}
$$

Applying Theorem 4.1 to the corrector at the point $t_{n}$ with $e_{n}=y_{n}-y_{\mathrm{ex}}\left(t_{n}\right)$ and assuming that $j b^{\mathrm{T}} c^{j-1}=1, j=1, \ldots, q$, yields

$$
\begin{aligned}
u_{n+1} & -y_{\mathrm{ex}}\left(t_{n+1}\right) \\
= & R(z)\left[y_{n}-y_{\mathrm{ex}}\left(t_{n}\right)\right] \\
& +\frac{1}{(q+1) !} z \boldsymbol{b}^{\mathrm{T}}[I-z A]^{-1}\left[\boldsymbol{c}^{q+1}-(q+1) A \boldsymbol{c}^{q}\right] h^{q+1} y_{\mathrm{ex}}^{(q+1)}\left(t_{n}\right)+\mathrm{O}\left(h^{q+2}\right) ;
\end{aligned}
$$

hence,

$$
\begin{aligned}
y_{n+1}-y_{\mathrm{ex}}\left(t_{n+1}\right) & =y_{n+1}-u_{n+1}+u_{n+1}-y_{\mathrm{ex}}\left(t_{n+1}\right) \\
& =y_{n+1}-u_{n+1}+R(z)\left[y_{n}-y_{\mathrm{ex}}\left(t_{n}\right)\right]+\mathrm{O}\left(h^{q+1}\right) .
\end{aligned}
$$

Thus, using $(4.6 \mathrm{a})$ we obtain

Now, sup holds for predictor identical

Theorem of PDIRK

where $q$ is corrector

This th function formally However are expec the stage

The fo

Corollary

$$
\begin{aligned}
& y_{n+1}-y_{\mathrm{ex}}\left(t_{n+1}\right) \\
& \quad=\left(R(z)-S_{m}(z)\right)\left[y_{n}-y_{\mathrm{ex}}\left(t_{n}\right)\right]-\sum_{j=2} Q_{m j}(z) h^{j} y_{\mathrm{ex}}^{(j)}\left(t_{n}\right)+\mathrm{O}\left(h^{q+1}\right) .
\end{aligned}
$$

The functions $Q_{m i}(z)$ will be called the error functions of the PDIRK method.

Finally, we show that the function $R(z)-S_{m}(z)$ is identical with the stability function $R_{m}$ of the PDIRK method. For that purpose, we consider the particular case where the inhomogeneous term $g$ vanishes. It is easily verified that we then may write

$$
y_{n+1}-y_{\mathrm{ex}}\left(t_{n+1}\right)=R_{m}(z)\left[y_{n}-y_{\mathrm{ex}}\left(t_{n}\right)\right]+\left(R_{m}(z)-e^{z}\right) y_{\mathrm{ex}}\left(t_{n}\right), \quad g=0 .
$$

where $c^{*}$

Proof. In

so that 
Now, suppose that the initial value $y_{0}$ tends to zero. Then, $y_{\mathrm{ex}}(t)$ also tends to zero. Since (4.8) holds for vanishing $g$ too, it follows that $R_{m}(z)=R(z)-S_{m}(z)$. Notice that in the case of the predictor (2.5) the functions $S_{m}(z)$ and $E_{m}(z)$ as defined in Theorem 3.4 are apparently identical. Thus, we have proved the following PDIRK analogue of Theorem 4.1:

Theorem 4.2. For one-step predictors possessing the expansion (4.3b) with $c^{*}=c_{1}^{*}$ the global error of PDIRK methods when applied to the test equation $y^{\prime}(t)=\lambda y(t)+g(t)$ satisfies the recursion

$$
\begin{aligned}
& y_{n+1}-y_{\mathrm{ex}}\left(t_{n+1}\right)=R_{m}(z)\left[y_{n}-y_{\mathrm{ex}}\left(t_{n}\right)\right]-\sum_{j=2} Q_{m j}(z) h^{j} y_{\mathrm{ex}}^{(j)}\left(t_{n}\right)+\mathrm{O}\left(h^{q+1}\right), \\
& R_{m}(z)=R(z)-S_{m}(z), \quad S_{m}(z):=\boldsymbol{b}^{\mathrm{T}} A^{-1} Z^{m}(z) \sum_{j=2} \boldsymbol{q}_{j}(z) z^{j}, \\
& Q_{m j}(z):=\boldsymbol{b}^{\mathrm{T}} A^{-1} Z^{m}(z) \boldsymbol{q}_{j}(z),
\end{aligned}
$$

where $q$ is the stage order of the corrector, and $R(z)$ and $R_{m}(z)$ are the stability functions of the corrector and the PDIRK method, respectively.

This theorem shows that the stage order of PDIRK methods is only one, unless the error function $Q_{m 2}(z)$ is identically zero for the $m$-value used. (This is not surprising because formally PDIRK methods are just DIRK methods which are known to have stage order one.) However, as all error functions $Q_{m j}(z)$ contain the factor $Z^{m}(z)$, their maximal values $\left|Q_{m j}\right|$ are expected to decrease rapidly with $m$ in any region of the left halfplane, so that effectively the stage order shown in actual computation is much higher.

The following corollary presents an explicit expression of $Q_{m j}$ for the predictor (2.5).

Corollary 4.3. For the predictor (2.5) the error functions are given by

$$
\begin{aligned}
& Q_{m j}(z):=\frac{1}{j !} \boldsymbol{b}^{\mathrm{T}} A^{-1} Z^{m}(z) z^{-1}\left(j \boldsymbol{c}^{j-1}-[I-z B]^{-1}\left[j\left(\boldsymbol{c}^{*}\right)^{j-1}-z\left(\boldsymbol{c}^{*}\right)^{j}\right]\right), \\
& j=2, \ldots, q
\end{aligned}
$$

where $c^{*}:=(B+E) \boldsymbol{e}$.

Proof. In the case (2.5) the expansion (4.3b) becomes

$$
\begin{aligned}
\boldsymbol{Y}^{(())} & =[I-z B]^{-1}\left([I+z E] y\left(t_{n}\right) \boldsymbol{e}+h E g\left(t_{n}\right) \boldsymbol{e}+h B g\left(t_{n} \boldsymbol{e}+h c^{*}\right)\right) \\
& =y\left(t_{n}\right) \boldsymbol{e}+[I-z B]^{-1}\left(E h y^{\prime}\left(t_{n}\right) \boldsymbol{e}+B \sum_{j=1} \frac{1}{j !} h^{j} y^{(j)}\left(t_{n}\right)\left[j\left(c^{*}\right)^{j-1}-z\left(c^{*}\right)^{j}\right]\right),
\end{aligned}
$$

so that

$$
\begin{aligned}
& c_{1}^{*}(z)=[I-z B]^{-1}\left(E e+B e-z B c^{*}\right)=c^{*}=(B+E) e, \\
& c_{j}^{*}(z)=[I-z B]^{-1} z B\left[j z^{-1}\left(c^{*}\right)^{j-1}-\left(c^{*}\right)^{j}\right], \quad j \geqslant 2 .
\end{aligned}
$$


By virtue of Theorem 4.2 we may write

$$
\begin{aligned}
Q_{m j}(z)= & \boldsymbol{b}^{\mathrm{T}} A^{-1} Z^{m}(z) \boldsymbol{q}_{j}(z) \\
= & \frac{1}{j !} \boldsymbol{b}^{\mathrm{T}} A^{-1} Z^{m}(z)\left[\boldsymbol{c}_{j}(z)-\boldsymbol{c}_{j}^{*}(z)+z^{-1} \gamma_{j}(z)\right] \\
= & \frac{1}{j !} \boldsymbol{b}^{\mathrm{T}} A^{-1} Z^{m}(z) z^{-1} \\
& \times\left([I-z A]^{-1}\left[j \boldsymbol{c}^{j-1}-z \boldsymbol{c}^{j}\right]-[I-z B]^{-1}\left[j\left(c^{*}\right)^{j-1}-z\left(c^{*}\right)^{j}\right]\right) .
\end{aligned}
$$

By means of the simplifying condition $C(q)$ associated with (2.1) (cf. [3]), we obtain the relation $j A c^{j-1}=c^{j}$ for $j=2, \ldots, q$ which leads to the result of the corollary.

\subsection{Last step-value predictor with constant iteration parameters}

In the case of the predictor LSP (predictor (2.5) with $B=E=O$ ) with constant iteration parameters $(D=\delta \cdot I)$, the error functions $Q_{m j}(z)$ can be factorized into factors that depend on $z$ and factors that do not depend on $z$. This enables us to derive an explicit upper bound for $Q_{m j}(z)$.

Theorem 4.4. Let $D=\delta \cdot I$ and let the predictor be given by (2.5). Then the error function bound in a region $\mathbb{R}$ is given by

$$
\begin{aligned}
& \left|Q_{m j}\right|_{\mathbb{H}}=\frac{1}{(j-1) !} d(m)\left|\boldsymbol{b}^{\mathrm{T}} A^{-1} D\left(D^{-1} A-I\right)^{m} \boldsymbol{c}^{j-1}\right|, \quad j=2, \ldots, q ; \\
& d(m):=\left|\frac{(\delta z)^{m-1}}{(1-\delta z)^{m}}\right|_{\mathbb{R}} .
\end{aligned}
$$

If $\mathbb{R}$ is the infinite wedge defined by

$$
W:=\{z: \pi / 2 \leqslant \phi \leqslant \arg (z) \leqslant \pi,-\pi \leqslant \arg (z) \leqslant-\phi\},
$$

then

$$
d(m)=\frac{x_{m}^{m-1}}{\left(m\left(1-x_{m} \cos (\phi)\right)\right)^{m / 2}},
$$

where $x_{m}$ is the positive root of the equation $x^{2}-(2-m) x \cos (\phi)-m+1=0$.

Proof. The expression for the error bound $\left|Q_{m j}\right|_{\mathbb{R}}$ immediately follows from Corollary 4.3. In order to derive an expression for the function $d(m)$ we first observe that

$$
\left|\frac{z}{1-z}\right|=\frac{|z|}{\sqrt{1-2|z| \cos (\arg (z))+|z|^{2}}},
$$


Table 4

Values of $\left|Q_{j}\right|_{\mathbb{R}}$ and $\left|Q_{m j}\right|_{\mathbb{B}}$ with $\mathbb{R}=\{z: \operatorname{Re} z \leqslant 0\}$ for the Nørsett-DIRK methods and fixed-number-of-iterations PDIRK methods

\begin{tabular}{lllllllll}
\hline Method/PC pair & $k$ & $\delta_{i}$ & $m$ & $p^{*}$ & $j=2$ & $j=3$ & $j=4$ & $j=5$ \\
\hline Nørsett-DIRK & 1 & & 2 & 3 & 0.144 & 0.076 & 0.024 & 0.0055 \\
\{LSP, Radau IIA\} & 2 & 0.43586650 & 3 & 3 & 0.024 & 0.015 & 0.005 & 0.0012 \\
\{LSP, Lagrange\} & 2 & 0.43586650 & 3 & 3 & 0.038 & 0.015 & 0.005 & 0.0012 \\
Nørsett-DIRK & 1 & & 3 & 4 & 0.112 & 0.054 & 0.015 & 0.0040 \\
\{LSP, Radau IIA\} & 3 & 0.278053841 & 5 & 5 & 0.019 & 0.006 & 0.0014 & 0.0003 \\
\{LSP, Lagrange\} & 3 & 0.572816063 & 4 & 4 & 0.046 & 0.013 & 0.0001 & 0.0012 \\
\{LSP, Lagrange\} & 4 & 0.278053841 & 5 & 5 & 0.025 & 0.005 & 0.0001 & 0.0001 \\
\hline
\end{tabular}

where $\pi / 2 \leqslant \arg (z) \leqslant \pi$ or $-\pi \leqslant \arg (z) \leqslant-\pi / 2$. Hence,

$$
\left|\frac{z^{m-1}}{(1-z)^{m}}\right|=\frac{|z|^{m-1}}{\left[1-2|z| \cos (\arg (z))+|z|^{2}\right]^{m / 2}} \text {. }
$$

Since the function $z^{m-1}(1-z)^{-m}$ is analytic, its maximum value in $\mathbb{W}$ is assumed at a point on the line $\arg (z)=\phi$. An elementary calculation reveals that the modulus of this point is given by the positive root $x_{m}$ of the quadratic equation $x^{2}-(2-m) x \cos (\phi)-m+1=0$. This leads us to the bound $d(m)$ given in the theorem.

This theorem shows that in the case where the relevant $z$-values are in an infinite wedge $\mathbb{W}$, the optimal choice of the matrix $D=\delta \cdot I$ does not depend on $\mathbb{W}$. Furthermore, the function $d(m)$ is slowly varying with $m$. This can be concluded from the extreme cases where $\mathbb{R}$ is either only the negative axis or the whole left halfplane. We then have, respectively, $x_{m}=m-1$ and $x_{m}=\sqrt{m-1}$, which yields

$$
d(m)=\frac{1}{m-1}\left(1-\frac{1}{m}\right)^{m} \text { and } d(m)=\frac{1}{\sqrt{m-1}}\left(1-\frac{1}{m}\right)^{m / 2} .
$$

Thus, within a few iterations the function $d(m)$ slowly converges to zero.

It is of interest to compare the error functions $Q_{j}(z)$ of conventional DIRK methods (cf. Theorem 4.1) with the error functions $Q_{m j}(z)$ of PDIRK methods. Table 4 presents a comparison for two conventional Nørsett-DIRK methods [9] and a few L-stable, fixed-numberof-iterations PDIRK methods constructed according to option 1 [12]. In this table, $k$ denotes the number of processors needed, $p^{*}$ is the order of the method, and $m$ denotes the number of sequential stages per step (both for the Nørsett-DIRK and PDIRK methods). Clearly, the PDIRK methods possess considerably smaller error bounds.

\subsection{Minimal-spectral-radius PDIRK methods}

Table 5 lists values of $\left|Q_{m j}\right|_{\mathbb{R}}$ with $\mathbb{R}=\{z: \operatorname{Re} z \leqslant 0\}$ for minimal-spectral-radius PDIRK methods (option 2), based on \{LSP, Radau IIA\} pairs and using the iteration parameters given 
Table 5

Values of the error constants for minimal-spectral-radius PDIRK methods

\begin{tabular}{llllllll}
\hline PC pair & $k$ & $m$ & $p^{*}$ & $j=2$ & $j=3$ & $j=4$ & $j=5$ \\
\hline LLSP, Radau IIA\} & 2 & 2 & 2 & 0.0249 & 0.0263 & 0.0102 & 0.0027 \\
& & 3 & 3 & 0.0060 & 0.0062 & 0.0024 & 0.0006 \\
\{LSP, Radau IIA\} & 3 & 3 & 3 & 0.0360 & 0.0086 & 0.0027 & 0.00076 \\
& & 4 & 4 & 0.0138 & 0.0031 & 0.0009 & 0.00025 \\
& & 5 & 5 & 0.0052 & 0.0012 & 0.0003 & 0.00009 \\
(LSP, Radau IIA\} & 4 & 5 & 5 & 0.0153 & 0.00098 & 0.000031 & 0.00004 \\
& & 6 & 6 & 0.0079 & 0.00051 & 0.000016 & 0.00002 \\
& & 7 & 7 & 0.0041 & 0.00027 & 0.000008 & 0.00001 \\
& & $\ldots$ & 7 & $r \approx 0.50$ & $r \approx 0.52$ & $r \approx 0.50$ & $r \approx 0.52$ \\
\hline
\end{tabular}

in (3.3). It turns out that for $m>p^{*}$ the error constants decrease by an almost constant reduction factor $r$ as $m$ increases by 1 and that they are substantially smaller than those of the fixed-number-of-iterations PDIRK methods of Table 4. (Notice that $r$ is almost independent of j.)

For future reference, we give a survey of the principal stiff error constants $\left|Q_{m 2}\right|_{\mathbb{B}}$ with $\mathbb{R}=\{z: \operatorname{Re} z \leqslant 0\}$ for a number of PC pairs. In Table $6, p$ denotes the order of the corrector and the order of the iterated method is in all cases given by $p^{*}=\min \{p, m\}$. From these results we conclude that the explicit predictor LSP leads to slightly smaller principal error constants than the implicit predictor IEP, provided that we count the application of IEP as an additional iteration. Furthermore, the Lagrange-based methods show considerably smaller error constants. However, we should bear in mind that the nonstiff error constants of the Radau-based methods decrease much faster than those of the Lagrange-based methods

Table 6

Values of the principal error constant for minimal-spectral-radius PDIRK methods

\begin{tabular}{lllllll}
\hline PC pair & $k$ & $p$ & $m=k$ & $m=k+1$ & $m=k+2$ & $r$ \\
\hline ILSP, Radau IIA & 2 & 3 & 0.025 & 0.0060 & 0.0015 & 0.25 \\
\{IEP, Radau IIA & 2 & 3 & 0.024 & 0.0059 & 0.0015 & 0.25 \\
\{LSP, Lagrange\} & 2 & 3 & 0.013 & 0.0023 & 0.0004 & 0.18 \\
\{IEP, Lagrange\} & 2 & 3 & 0.006 & 0.0011 & 0.0002 & 0.18 \\
\{LSP, Radau IIA & 3 & 5 & 0.036 & 0.0138 & 0.0052 & 0.40 \\
\{IEP, Radau IIA & 3 & 5 & 0.014 & 0.0053 & 0.0020 & 0.41 \\
\{LSP, Lagrange\} & 3 & 4 & 0.008 & 0.0034 & 0.0014 & 0.40 \\
IIEP, Lagrange\} & 3 & 4 & 0.004 & 0.0018 & 0.0007 & 0.40 \\
\{LSP, Radau IIA\} & 4 & 7 & 0.027 & 0.0153 & 0.0079 & 0.50 \\
\{IEP, Radau IIA\} & 4 & 7 & 0.017 & 0.0088 & 0.0044 & 0.50 \\
\{LSP, Lagrange & 4 & 5 & 0.022 & 0.0092 & 0.0037 & 0.40 \\
\{IEP, Lagrange\} & 4 & 5 & 0.013 & 0.0054 & 0.0021 & 0.40 \\
\hline
\end{tabular}


because of the high (nonstiff) orders of the Radau correctors. Finally, note that the reduction factors are very close to the $\|C\|$-values listed in Table 1.

\section{Concluding remarks}

In this paper, we have studied special characteristics, such as the rate of convergence, the (linear) stability, and the stiff error constants, of PDIRK methods based on Radau IIA and Lagrange correctors using various types of iteration parameters and predictors. The minimalspectral-radius methods turn out to be either comparable or superior to fixed-number-of-iterations methods. Confining our considerations to minimal-spectral-radius methods, the following conclusions can be drawn from our analysis:

- Rate of convergence: Lagrange correctors are superior to Radau correctors for $k=2$ or $k=4$. For $k=3$, these correctors are comparable.

- Linear stability: Lagrange correctors are slightly superior to Radau correctors. The implicit predictors IEP and BDP are superior to the explicit predictors EXP and LSP.

- Order reduction: Lagrange correctors are superior to Radau correctors (both with respect to the stage order and the magnitude of the error constants). The explicit predictor LSP is slightly superior to the implicit predictor IEP.

- Nonstiff error constants: The two-stage Radau corrector is comparable with the two-stage Lagrange corrector. Radau correctors are by far superior to Lagrange correctors for $k>2$. The predictors EXP and BDP are by far superior to the predictors LSP and IEP.

By these conclusions, we are led to recommend PDIRK methods using an \{EXP, Radau\} PC pair and the minimal-spectral-radius iteration strategy as the most efficient in the class of PDIRK methods.

\section{References}

[1] K. Burrage, W.H. Hundsdorfer and J.G. Venwer, A study of B-convergence of Runge-Kutta methods, Computing 36 (1986) 17-34.

[2] J.C. Butcher, Implicit Runge-Kutta processes, Math. Comp. 18 (1964) $50-64$.

[3] J.C. Butcher, The Numerical Analysis of Ordinary Differential Equations: Runge-Kutta and General Linear Methods (Wiley, New York, 1987).

[4] K. Dekker and J.G. Verwer, Stability of Runge-Kutta Methods for Stiff Nonlinear Differential Equations (North-Holland, Amsterdam, 1984).

[5] E. Hairer, S.P. Nørsett and G. Wanner, Solving Ordinary Differential Equations, I: Nonstiff Problems, Springer Series in Computational Mathematics 8 (Springer, Berlin, 1987).

[6] E. Hairer and G. Wanner, Solcing Ordinary Differential Equations, II: Stiff and Differential-Algehraic Problems, Springer Series in Computational Mathematics 14 (Springer, Berlin, 1991).

[7] K.R. Jackson and S.P. Nørsett, The potential for parallelism in Runge-Kutta methods, Part 1: RK formulas in standard form, Tech. Rept. No. 239/90, Department of Computer Science, University of Toronto, Toronto, Ont. (1990).

[8] K.R. Jackson and S.P. Nørsett, The potential for parallelism in Runge-Kutta methods, Part 2: RK predictorcorrector formulas (in preparation). 
[9] S.P. Nørsett, Semi-explicit Runge-Kutta methods, Mathematics and Computation Rept. No. 6/74, Department of Mathematics, University of Trondheim, Trondheim, Norway (1974).

[10] L.F. Shampine, Implementation of implicit formulas for the solution of ODEs, SIAM J. Sci. Statist. Comput. 1 (1980) $103-118$.

[11] P.J. van der Houwen and B.P. Sommeijer, Iterated Runge-Kutta methods on parallel computers, SLAM J. Sci. Statist. Comput. 12 (1991) 1000-1028.

[12] P.J. van der Houwen, B.P. Sommeijer and W. Couzy, Embedded diagonally implicit Runge-Kutta algorithms on parallel computers, Math. Comp. 58 (1992) 135-159.

[13] A. Wolfbrandt, A study of Rosenbrock processes with respect to order conditions and stiff stability, Ph.D. Thesis, Chalmers University of Technology, Göteborg. Sweden (1977). 